\title{
Deformation mechanism of cryorolled Fe-36\%Ni strip steel
}

\author{
Changsheng $\mathrm{Li}^{\mathrm{a}}$, Jianjun Zheng, Yanlei Song, Shuai He, and Liqing Chen \\ State Key Laboratory of Rolling and Automation, Northeastern University, Shenyang 110819, \\ PR China
}

\begin{abstract}
The deformation mechanism of cryogenic rolled Fe-36\% Ni steel is investigated with rolling strain of $\varepsilon=0.24-0.92$. Experimental results show that both dislocation slip and twinning are activated at cryogenic temperature as strain $\varepsilon=0.24$, and the mean thickness of deformation twins is about $200 \mathrm{~nm}$. When the rolling strain increases to 0.53 , the mean thickness of deformation twins reduces to $50 \mathrm{~nm}$ and some curved deformation twins exists. As the rolling strain increases to 0.92 , the thickness of deformation twins is no longer changed and the micro shear bands exist due to the concentration of work hardening, which suggests that twinning is activated at the early stage of cryogenic rolling (CR) process. It is assumed that more deformation twins will be fragmented by shear bands during the following CR. The dislocation slip becomes the dominated mechanism again during the following deformation. The mechanism of the Fe-36\% Ni steel following CR was analyzed quantitatively.
\end{abstract}

\section{Introduction}

$\mathrm{Fe}-36 \% \mathrm{Ni}$ steel is world famous due to its excellent low expansion properties and widely used in various fields such as precision instrument, liquid natural gas (LNG) containers and core wires of long distance power cables [1]. So far, the plastic deformation behaviour, formation mechanism of the cube texture during cold rolling and work hardening rate of $\mathrm{Fe}-36 \% \mathrm{Ni}$ steel have been well understood [2-4]. The microstructure and texture studies have been carried out by traditional cold rolling, asymmetrical rolling and accumulative roll bonding. Zaefferer et al. [3] found that Fe-36\%Ni steel shows little recovery during cold rolling, and the cube recrystallization texture is observed after the high cold rolling due to cube nuclei develop significantly quicker than other orientations. By $83 \%$ thickness reduction asymmetrical rolling, the main deformed texture of $\mathrm{Fe}-36 \mathrm{Ni} \%$ steel is copper texture with a little rotation around the transverse direction axis, which revealed the formation of shear texture during asymmetrical rolling [5]. Up to now, the literature about $\mathrm{Fe}-36 \mathrm{Ni} \%$ steel deformed at cryogenic temperature (the low temperature is maintained by liquid nitrogen) is limited. Flor et al. [2] reported the deformation behaviour of $\mathrm{Fe}-35 \% \mathrm{Ni}$ at $77 \mathrm{~K}$ by tensile machine, in the same way, tensile tests was carried out to study the temperature and strain rate dependent mechanical properties of $\mathrm{Fe}-36 \mathrm{Ni} \%$ steel [6]. However, little information about microstructure evolution and deformation mechanism is involved in the two

\footnotetext{
${ }^{a}$ Corresponding author: lics@ral.neu.edu.cn
}

This is an Open Access article distributed under the terms of the Creative Commons Attribution License 4.0, which permits unrestricted use, distribution, and reproduction in any medium, provided the original work is properly cited. 


\section{MATEC Web of Conferences}

papers mentioned above. Fe-36\% Ni steel is similar to $\mathrm{Cu}$ with medium stack fault energy (SFE) and face-centered cubic (FCC) structure at the room temperature [7].

As we know that the deformation mechanism is dominated by dislocation slip for medium SFE metal at ambient temperature [8], while SFE will be lower with the decreased temperature, so there may be a significant change on deformation mechanism of $\mathrm{Fe}-36 \% \mathrm{Ni}$ steel during cryogenic rolling (CR). Indeed, CR can suppress dynamic recovery more effectively, resulting in a high density of defects and consequent grain refinement [9-11]. In addition, compared to some sever plastic deformation and conventional rolling, CR requires relatively low plastic deformation strains due to less friction and induces large strain [12]. In order to explore whether CR is an effective way to enhance the mechanical property of $\mathrm{Fe}-36 \% \mathrm{Ni}$ steel, it is essential to research the the deformation mechanism of Fe-36\% Ni steel during CR. In this paper, the deformation mechanism of cryorolled Fe-36\%Ni steel was investigated.

\section{Experimental}

The chemical composition of the steel used in this study is (wt. $\%$ ) $36.6 \mathrm{Ni}, 0.003 \mathrm{C}, 0.03 \mathrm{Si}, 0.02 \mathrm{Mn}$, $0.01 \mathrm{P}, 0.001 \mathrm{~S}, 0.12 \mathrm{Al}$ and balance Fe. The hot rolled sample was annealed at $1423 \mathrm{~K}$ for $1 \mathrm{~h}$ and water quenched to obtain the uniform austenitic microstructure, after that cut into plate samples with size of $100 \mathrm{~mm} \times 40 \mathrm{~mm} \times 5 \mathrm{~mm}$ by electric discharge machining. Subsequently, the plates were rolled to different thickness at cryogenic temperature. The rolling was carried out on a $\phi 160 \mathrm{~mm}$ laboratory mill and the rolling speed was $0.04 \mathrm{~m} / \mathrm{s}$. During cryogenic deformation, samples were dipped in liquid nitrogen at least $10 \mathrm{~min}$ before and after each rolling pass, with a reduction less than $15 \%$ per pass. The deformation strain is defined as $\varepsilon=\ln \left(H_{0} / H_{1}\right)$, where $H_{0}$ and $H_{1}$ are the initial and final thickness of the sample respectively. The temperature of samples was estimated to be below $173 \mathrm{~K}$ during every process. Samples cut in longitudinal direction were taken at different strains for study of microstructure.

Microstructure characterization was performed on a Leica DMIRM optical microscope (OM) and FEI Tecnai $G^{2}$ F20 transmission electron microscope (TEM) respectively. Samples for optical microscopy were polished and etched with 30 vol. $\% \mathrm{HNO}_{3}$ solution. For TEM, the electro-polishing was carried out with $10 \mathrm{vol} . \% \mathrm{HClO}_{4}$ and $90 \mathrm{vol} . \% \mathrm{CH}_{3} \mathrm{COOH}$ solution at $263 \mathrm{~K}$ and a potential of $20 \mathrm{~V}$. The mean thickness of deformation twins was measured by the linear intercept method followed from 10 TEM images.

\section{Results and discussion}

Figure 1a shows the microstructure of initial specimen, it was consisted of equiaxed grain, and the mean grain size was about $150 \mu \mathrm{m}$. The grains contained a certain amount of annealing twins and no interal subtructure was frequently observed. Microstructure of CR samples with different strain were presented in Fig. 1b-d. It can be seen that there were two different regions in Fig. $1 b(\varepsilon=0.24)$. Region I (black solid circle in Fig. 1b) was similar to that of initial grains, however region II (white solid circle in Fig. 1b) consisted of some parallel deformation bands, which implied that deformation inside sample was heterogeneous. Figure $2 a$ and Fig. $2 b$ was the TEM images of microstructure corresponding to the regions mentioned above. Figure 2a showed that a large amount of dislocations in region I, also some hundred nanometers dislocation cells could be found. This indicated that only the deformation mechanism of dislocation slip was activated in this region. The bands embedded in region II were proved to be deformation twins (Fig. 2b). The corresponding selected diffraction pattern along a [011] zone axis revealed that the deformation twins followed the $\{111\}<112>$ relationship, and the mean thickness was about $200 \mathrm{~nm}$.

Ordinarily, deformation mechanisms are dependent on the value of SFE. For Fe-36\%Ni steel with the SFE of $122 \mathrm{~mJ} \mathrm{~m}^{-2}$ [13], dislocation slip is the common deformation mechanism informed from the previous studies [3], and the information about mechanical twinning is limited. It is well understood that 

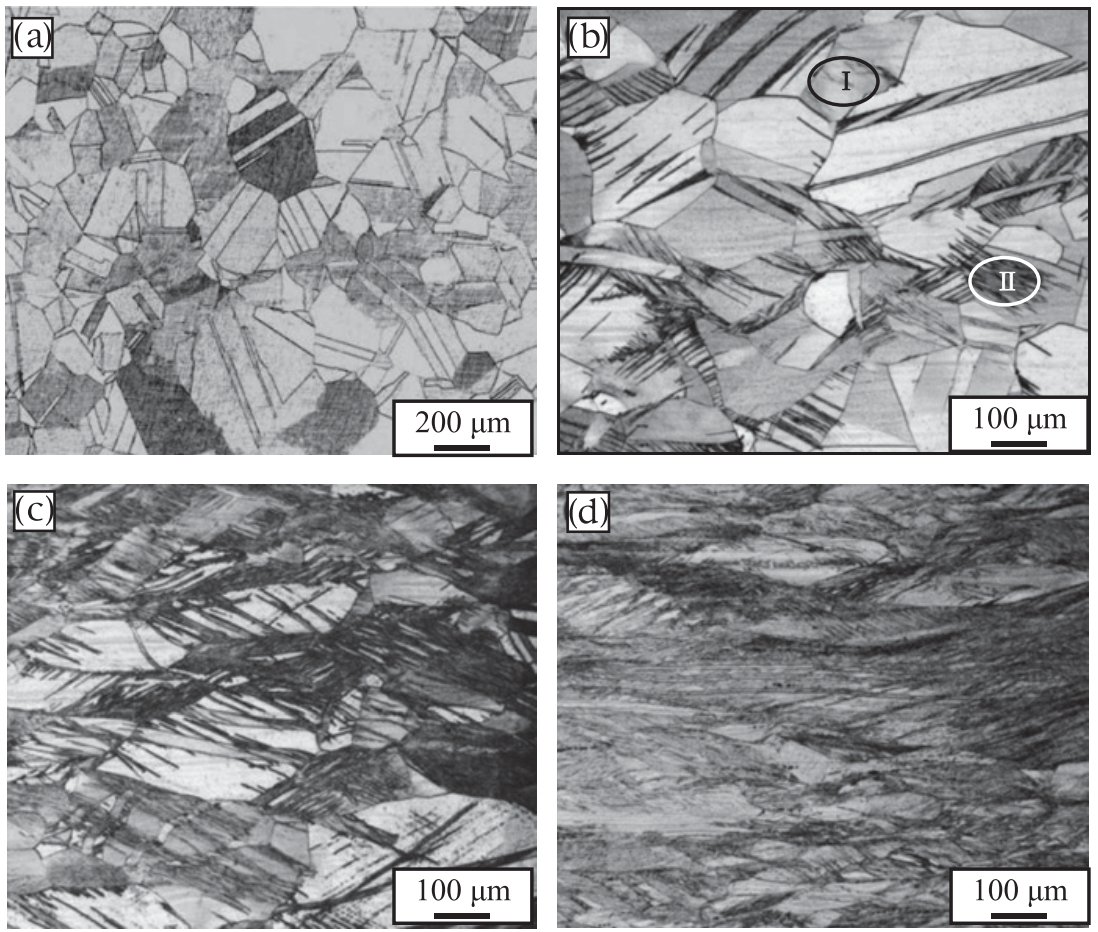

Figure 1. Microstructures of initial sample (a) and CR Fe-36\% Ni steel with different strains, (b) $\varepsilon=0.24$, (c) $\varepsilon=0.53$, (d) $\varepsilon=0.92$.

twinning is the main deformation mechanism in materials with low SFE, such as $\mathrm{Cu}-\mathrm{Al}$ alloy, twinninginduced plasticity (TWIP) steels [14]. In this study, SFE of Fe-36\%Ni steel decreases when deformation is carried out at cryogenic temperature. Lowering of SFE can result in the inhibition of dislocation cross slip and the increase of partial dislocation density. In FCC metals, passage of partial dislocations leads to twinning during rolling process [15]. In theory, the critical resolved shear stress (CRSS) for twinning is less depending on temperature compared to that for slip. With decreased temperature, the difference between the values of the CRSS for twinning and slip will decrease [16]. At cryogenic temperature, the difference mentioned above is much smaller, hence the mechanism of twinning can be activated during CR. Some grains are divided by deformation twins due to the orientation is preferred to induce twinning (Fig. 2b), grains with other orientations coordinate the deformation process by intragranular cross-slip of dislocations (Fig. 2a). Therefore the deformation mechanism is dislocation coexist twinning when $\varepsilon<0.24$.

With the increasing deformation strain $\varepsilon=0.53$, it can be seen that the deformation twins almost presented in each original equiaxed grains (Fig. 1c). Obviously, the deformation twin density increased and the mean thickness reduced to $50 \mathrm{~nm}$ as shown in Fig. 2c. Besides some deformation twins became curved as a result of rotation to the rolling direction (dotted circle in Fig. 2c). As the CRSS for twinning can be achieved in plenty of strain hardening grains, more grains were divided by deformation twins owing to the increased partial dislocation density. The reduction of deformation twin thickness reveals that new twins formed inside the primary deformation twins. Hence twinning becomes the dominated deformation mechanism during strain $(0.24<\varepsilon<0.53)$. Besides, the appearance of curved deformation twins due to the interaction between dislocations and twin boundaries proved that twinning is initially suppressed with this strain. For the sample with strain $\varepsilon=0.92$, microstructure of deformation matrix was seem to be a little blurry, and it was hard to distinguish the primary deformation twins. TEM 

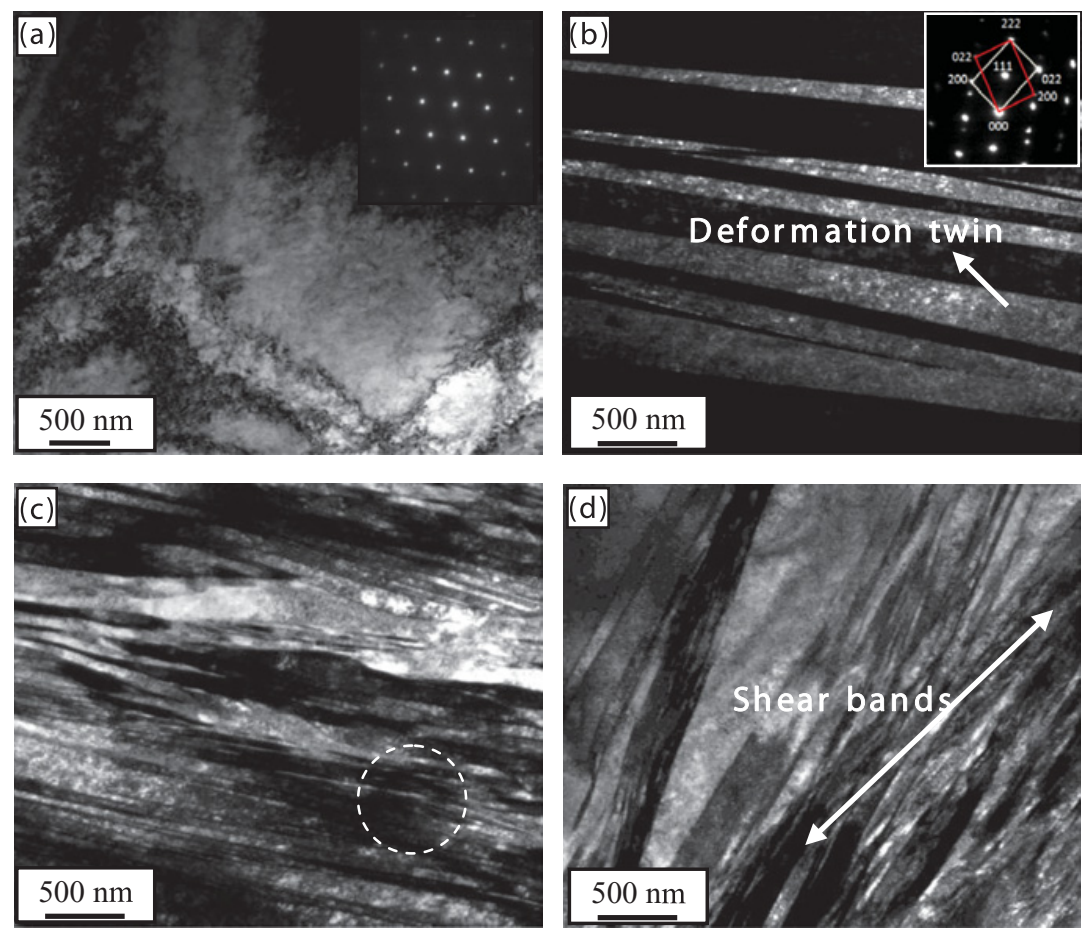

Figure 2. TEM images for CR Fe-36\% Ni steel with different strains (a) $\varepsilon=0.24$ (Fig. 1b region I), (b) $\varepsilon=0.24$ (Fig. 1b region II), (c) $\varepsilon=0.53$, (d) $\varepsilon=0.92$.

statistical results indicated that the thickness and destiny of deformation twins were no longer changed. This was in accord with the forementioned result that twinning was restrained. In addition, a specific feature of the microstructural evolution was the appearance of micro shear bands as shown in Fig. $2 \mathrm{~d}$. They intersected the deformation twins and formed a certain angle. It indicated that the previous deformation twins tended to rearrange along the rolling direction. Shear banding is a typical low temperature phenomenon which often emerges when homogeneous plastic deformation cannot sustain work hardening [17]. At this strain deformation twins are so thin that it is different to activate twining to be refined, twinning was suppressed due to the higher work hardening. Then shear bands existed and the deformation twins were gradually fragmented into small structures with following deformation (Fig. 2d). As a result slip became the dominated deformation mechanism again. This corresponds to other studies that the formation of micro shear bands in FCC metals is due to the highly concentration of plastic strain $[14,18]$.

In summary, twinning is only activated at the early deformation stage with rolling strain up to 0.53 . In this paper, the whole deformed process obeys the mechanism of slip $\rightarrow$ twinning $\rightarrow$ slip. In subsequent deformation, more deformation twins will be fragmented by shear bands during the following CR, and the original equiaxed grains will be refined. The deformation model is same as the one at room temperature depending on various systems of dislocation slip.

\section{Conclusions}

Fe-36\% Ni steel undergoes predominantly dislocation slip during room temperature deformation, but slip plus twinning during cryogenic deformation process. The cryogenic temperature results in significant change in the deformation mechanism of Fe-36\%Ni steel. Due to the complex deformation mechanisms 


\section{ICNFT 2015}

during the process of $\mathrm{CR}, \mathrm{Fe}-36 \% \mathrm{Ni}$ exhibits good ductility without fracture existence even cryogenic rolled at large strains. The whole deformed process obeys the mechanism of slip $\rightarrow$ twinning $\rightarrow$ slip.

The financial support of National Natural Science Foundation of China (51174057, 51274062), the National High Technology Research and Development Program (2012AA03A503) and Research Fund for the Doctoral Program of Higher Education of China (20130042110040) are greatly acknowledged.

\section{References}

[1] M. Shiga, Curr. Opin. Solid State Mater. Sci. 1, 340 (1996)

[2] H. Flor, H.J. Gudladt, Ch. Schwink, Acta Metall. 28, 1611 (1980)

[3] S. Zaefferer, T. Baudin, R. Penelle, Acta Mater. 49, 1105 (2001)

[4] A.E. Ekinci, N. UÇar, G. Çankaya, B. Düzgün, Phys. Stat. Sol. 191, 387 (2002)

[5] D. Solas, S. Chhann, T. Baudin, R. Penelle, Mater. Sci. Forum 636-637, 538 (2010)

[6] W.S. Park, M.S. Chun, M.S. Han, M.H. Kim, J.M. Lee, Mater. Sci. Eng. A 528, 5790 (2011)

[7] B.L. Li, N. Tsuji, N. Kamikawa, Mater. Sci. Eng. A 423, 331 (2006)

[8] V.S. Sarma, J. Wang, W.W. Jian, A. Kauffmann, H. Conrad, J. Freudenberger, Y.T. Zhu, Mater. Sci. Eng. A 527, 7624 (2010)

[9] Y. Wang, M. Chen, F. Zhou, E. Ma, Nature 419, 912 (2002)

[10] T. Shanmugasundaram, B.S. Murty, V.S. Sarma, Scr. Mater. 54, 2013 (2006)

[11] S.V. Zherebtsov, G.S. Dyakonov, A.A. Salem, Acta Mater. 61, 1167 (2013)

[12] N. Rangaraju, T. Raghuram, B.V. Krishna, Mater. Sci. Eng. A 398, 246 (2005)

[13] K. Tirsatine, H. Azzeddine, T. Baudin, A.L. Helbert, F. Brisset, B. Alili, D. Bradai, J. Alloy. Compd. 610, 352 (2014)

[14] P. Kusakin, A. Belyakov, C. Haase, R. Kaibyshev, D.A. Molodov, Mater. Sci. Eng. A 617, 52 (2014)

[15] Y.T. Zhu, X.L. Wu, X.L. Liao, J. Narayan, S.N. Mathaudhu, L.J. Kecskes, Appl. Phys. Lett. 95, 031909 (2009)

[16] S.V. Zherebtsov, G.S. Dyakonov, A.A. Salem, V.I. Sokolenko, G.A. Salishchev, S.L. Semiatin, Acta Mater. 61, 1167 (2013)

[17] Q. Wei, D. Jia, E. Ma, K.T. Ramesh, Appl. Phys. Lett. 81, 1240 (2002)

[18] Y.S. Li, N.R. Tao, K. Lu, Acta Mater. 56, 230 (2008) 